\title{
ON AN INEQUALITY RELATING TO SUM SETS
}

\author{
C. E. M. PEARCE ${ }^{1}$ and J. E. PEČARIĆ ${ }^{2}$
}

(Received 17 November 1993; accepted 14 April 1994)

\begin{abstract}
We show how a short and elementary proof can be provided for a recently-published inequality ([6], [4]) which has found a number of applications.
\end{abstract}

\section{Introduction}

Probability-measure inequalities for sum sets have found a number of applications, see, for example, Brown and Williamson [3] for a coin-tossing application and Newhouse [7] and Palis and Takens [8] in connection with dynamical systems. They are often also intimately associated with combinatorial counting problems, as is the case in the present context.

In 1974, G. Brown and W. Moran [1] showed that a key probability inequality for uncountable sum sets could be deduced if a related counting inequality held for certain discrete sum sets, and that this in turn would follow from the truth of the inequality

$$
x^{\alpha} y^{\alpha}+\max \left[x^{\alpha}(1-y)^{\alpha}, y^{\alpha}(1-x)^{\alpha}\right]+(1-x)^{\alpha}(1-y)^{\alpha} \geq 1
$$

for $0 \leq x, y \leq 1$ and $\alpha=\log _{4} 3$. Brown and Moran were unable to establish (1) at the time. Quite a rich literature, an historical perspective on some of which is recounted by Brown in [2], has developed around both (1) and the original problem. Relation (1) possesses an $m$-variable generalization

$$
\prod_{i=1}^{m} x_{i}^{\alpha}+\sum_{j=1}^{m-1} \max _{\pi} \prod_{i=1}^{j} x_{\pi(i)}^{\alpha} \prod_{i=j+1}^{m}\left(1-x_{\pi(i)}\right)^{\alpha}+\prod_{i=1}^{m}\left(1-x_{i}\right)^{\alpha} \geq 1,
$$

where $\pi$ denotes a permutation of $\{1, \ldots, m\}$ and

$$
\alpha=\alpha(m)=\frac{\log (m+1)}{m \log 2} .
$$

\footnotetext{
'Department of Applied Mathematics, The University of Adelaide, Australia 5005.

${ }^{2}$ Faculty of Textile Technology, University of Zagreb, Zagreb, Croatia.

(C) Australian Mathematical Society, 1995, Serial-fee code 0334-2700/95
} 
If the $x_{i}$ are ordered by

$$
1 \geq x_{1} \geq \ldots \geq x_{m} \geq 0,
$$

this may be expressed more simply as

$$
f\left(x_{1}, \ldots, x_{m}\right) \geq 1,
$$

where

$$
f\left(x_{1}, \ldots, x_{m}\right) \equiv x_{1}^{\alpha} \ldots x_{m}^{\alpha}+x_{1} \ldots x_{m-1}^{\alpha}\left(1-x_{m}\right)^{\alpha}+\ldots+\left(1-x_{1}\right)^{\alpha} \ldots\left(1-x_{m}\right)^{\alpha} .
$$

It was established by Landau, Logan and Shepp [6] and independently by Brown, Keane, Moran and Pearce [4].

This apparently new and superficially inoccuous inequality turned out to be quite tricky to prove. As noted in [6]: "since the inequality seems to be a matter of real variables, it is perhaps surprising that our proof is based on conformal mapping and Hadamard's three-circle theorem." The proof of [4] is more elementary.

In fact, in [6] the following extension of (3) is proved.

Suppose $\alpha>0$ and $1 \geq x_{1} \geq \ldots \geq x_{m} \geq 0$ and let $f$ be defined by (4). Then

$$
f\left(x_{1}, \ldots, x_{m}\right) \geq \min \left[1,(m+1) 2^{-m \alpha}\right] .
$$

Here we shall note that we can use the simple method of [4] to obtain the general inequality (5). We show also how the argument of [4] may be shortened considerably.

\section{Results}

The following lemma was proved in [4].

LEMMA 1. Denote by $w(m) \geq 0$ the infimum of $f$, so that

$$
f\left(x_{1}, \ldots, x_{m}\right) \geq w(m) .
$$

If the values $x_{1}, \ldots, x_{m}$ are such that $f\left(x_{1}, \ldots, x_{m}\right)=w(m)$, then $x_{1}=\ldots=x_{m}=x$, say.

Now we have the following lemma, which extends Lemma 3 of [4].

LEMMA 2. (a) For $\alpha=\alpha(m)$,

$$
\frac{\sinh (m+1) \alpha t}{(\sinh \alpha t) \cosh ^{m \alpha} t} \geq m+1 \quad(t \geq 0)
$$


(b) for all $\beta$ satisfying $0 \leq \beta \leq \alpha(m)$

$$
1+b^{\beta}+b^{2 \beta}+\ldots+b^{m \beta} \geq(1+b)^{m \beta} \quad(b \geq 0) ;
$$

(c) for all $\beta \geq \alpha(m)$

$$
1+b^{\beta}+b^{2 \beta}+\ldots+b^{m \beta} \geq(m+1) 2^{-m \beta}(1+b)^{m \beta} \quad(b \geq 0) .
$$

Proof. Parts (a) and (b) are established in [4], while for $\beta \geq \alpha(m)$ we have

$$
\begin{aligned}
1+b^{\beta}+b^{2 \beta}+\ldots+b^{m \beta} & =1+\left(b^{\beta / \alpha(m)}\right)^{\alpha(m)}+\ldots+\left(b^{\beta / \alpha(m)}\right)^{m \alpha(m)} \\
& \geq\left[1+b^{\beta / \alpha(m)}\right]^{m \alpha(m)} \quad \text { by }(7) \\
& \geq 2^{m(\alpha(m)-\beta)}(1+b)^{m \beta} \quad \text { by Jensen's inequality } \\
& =(m+1) 2^{-m \beta}(1+b)^{m \beta} .
\end{aligned}
$$

From (7) and (8) we can formulate the following result.

If $\alpha>0$, then

$$
1+b^{\alpha}+b^{2 \alpha}+\ldots+b^{m \alpha} \geq \min \left[1,(m+1) 2^{-m \alpha}\right](1+b)^{m \alpha} .
$$

With the substitutions $x=1 /(1+b), 1-x=b /(1+b)$, (5) now follows from Lemma 1 and (9).

REMARK. As observed in [4], (6) and (7) are equivalent. However (6) arises as a special case of a theorem of Pittenger [9] (see Bullen, Mitrinović and Vasić [5, Theorem 5, page 349] for a more accessible account). For $r>0$, Pittenger's theorem gives in particular that (modulo an obvious misprint in [5])

$$
\left(\cosh r_{1} t\right)^{1 / r_{1}} \leq\left[\frac{\sinh (r+1) t}{(r+1) \sinh t}\right]^{1 / r} \leq\left(\cosh r_{2} t\right)^{1 / r_{2}},
$$

where

$$
\begin{aligned}
& r_{1}=\min \left[\frac{r+2}{3}, \frac{r \log 2}{\log (r+1)}\right], \\
& r_{2}=\max \left[\frac{r+2}{3}, \frac{r \log 2}{\log (r+1)}\right] .
\end{aligned}
$$

Replace $t$ by $\alpha t$. Since

$$
r_{1}=r \log 2 / \log (r+1)
$$

for $r \geq 1$, we have $r / r_{1}=r \alpha(r)$ and $r_{1} \alpha(r)=1$, and (6) follows at once from the left-hand relation of (10). This enables the end result of Lemmas 2 and 3 of [4] to be deduced directly, thereby shortening considerably the argument of [4] to provide a conveniently short proof of (2) and (5). 


\section{References}

[1] G. Brown and W. Moran, L.M.S. Research Symposium on Functional Analysis and Stochastic Processes, Durham, August 1974.

[2] G. Brown, "Some inequalities that arise in measure theory", J. Austral. Math. Soc. Ser. A 45 (1988) 83-94.

[3] G. Brown and J. H. Williamson, "Coin-tossing and sum sets", J. Austral. Math. Soc. Ser. A 43 (1987) 211-219.

[4] G. Brown, M. S. Keane, W. Moran and C. E. M. Pearce, "An inequality, with applications to Cantor measures and normal numbers", Mathematika 35 (1988) 87-94.

[5] P. S. Bullen, D. S. Mitrinović and P. M. Vasić, Means and their inequalities (D. Reidel, Dordrecht, 1988).

[6] H. J. Landau, B. F. Logan and L. A. Shepp, "An inequality conjectured by Hajela and Seymour arising in combinatorial geometry", Combinatorica 5 (1985) 337-342.

[7] S. E. Newhouse, "The abundance of wild hyperbolic sets and non-smooth stable sets for diffeomorphisms", Publ. Inst. des Hautes Etudes Scient. Publ. Math. 50 (1979) 101-151.

[8] J. Palis and F. Takens, "Cycles and measure of bifurcation sets for two-dimensional diffeomorphisms", Invent. Math. 82 (1985) 397-422.

[9] A. O. Pittenger, "Inequalities between symmetric, logarithmic and power means", Univ. Beograd. Elektrotehn. Fak. Ser. Mat. Fiz. 678-715 (1980) 19-23. 